\title{
ON CHUNG'S LAW OF LARGE NUMBERS ON SIMPLY CONNECTED STEP 2-NILPOTENT LIE GROUPS
}

\section{Neuenschwander ${ }^{1}$}

Ordóñez Cabrera and Sung (2002) have proved that under certain "moment" conditions, for triangular arrays of weighted Banach-valued random variables, a.s. convergence, convergence in $L^{1}$, convergence in probability, and complete convergence to 0 are equivalent, thus giving a variant of Chung's law of large numbers. We extend their result (under slightly sharper technical conditions) to symmetric random variables on simply connected step 2-nilpotent Lie groups.

\section{Introduction}

Ordóñez Cabrera and Sung [2] have given the following result (which is in some sense a generalization of Chung's law of large numbers). Let $B$ be a real separable Banach space. Assume $\left\{X_{n, i}\right\}_{n \geqslant 1,1 \leqslant i \leqslant k_{n}}$ (where $k_{n} \rightarrow \infty$ as $n \rightarrow \infty)$ is a triangular array of rowwise independent $B$-valued random variables and $\left\{a_{n, i}\right\}_{n \geqslant 1,1 \leqslant i \leqslant k_{n}} \subset \mathbb{R} \backslash\{0\}$ a triangular array of non-zero real constants such that $\max _{1 \leqslant i \leqslant k_{n}}\left|a_{n, i}\right| \rightarrow 0$ as $n \rightarrow \infty$. Let $\Psi$ be an even, positive, and continuous function and assume that the following "moment conditions" hold:

$$
\sum_{n=1}^{\infty} \sum_{i=1}^{k_{n}} \frac{\mathrm{E}\left(\Psi\left(|| X_{n, i}||\right)\right)}{\Psi\left(\left|a_{n, i}\right|^{-1}\right)}<\infty
$$

and

$$
\sum_{n=1}^{\infty}\left(\sum_{i=1}^{k_{n}} a_{n, i}^{2} \mathrm{E}\left\|X_{n, i}\right\|^{2}\right)^{s}<\infty
$$

for some $s>0$. Then convergence of $\sum_{i=1}^{k_{n}} a_{n, i} X_{n, i} \rightarrow 0, n \rightarrow \infty$ a.s., in $L^{1}$, completely, and in probability are all equivalent. (Recall that a sequence $\left\{Z_{n}\right\}_{n \geqslant 1}$ of $B$-valued random variables is said to converge completely to 0 if for every $\varepsilon>0$ we have

$$
\sum_{n=1}^{\infty} \mathrm{P}\left(\left\|Z_{n}\right\|>\varepsilon\right)<\infty
$$

The goal of this note is to extend the above result (under a mild additional "moment condition") to symmetric random variables on simply connected step 2-nilpotent Lie groups. These are the groups arising as follows: Let $[\cdot, \cdot]: \mathbb{R}^{d} \times \mathbb{R}^{d} \rightarrow \mathbb{R}^{d}$ be a bilinear map such that $[[\cdot, \cdot], \cdot] \equiv 0$. Then $G \cong \mathbb{R}^{d}$, equipped with the multiplication

$$
x \cdot y=x+y+\frac{1}{2}[x, y]
$$

is a simply connected step 2-nilpotent Lie group. The most prominent example is the Heisenberg group, which can be considered as the three-dimensional Lie algebra generated by the identity operator, the location operator, and the momentum operator. See e.g. [1] for more background information. Of course, on $G$ the inverse element of $x \in G$ is given by $(-x)$. A random variable $X$ is called symmetric if $X$ and $(-X)$ have the same distribution.

Let $C$ always denote a generic positive constant.

\section{Results}

Theorem 1. Let $G$ be a simply connected step 2-nilpotent Lie group. Assume $\left\{X_{n, i}\right\}_{n \geqslant 1,1 \leqslant i \leqslant k_{n}}$ (where $k_{n} \rightarrow \infty$ as $\left.n \rightarrow \infty\right)$ is a triangular array of rowwise independent symmetric $G$-valued random variables and

\footnotetext{
${ }^{1}$ Université de Lausanne, Institut des Sciences Actuarielles, CH-1015 Lausanne, Switzerland, Universität Bern, Switzerland, Institut für mathematische Statistik und Versicherungslehre, Sidlerstrasse 5, CH-3012 Bern, Federal Section of Cryptology, Ministery of Defense, CH-3003 Bern, Switzerland, e-mail: daniel.neuenschwander@bluewin.ch
}

Proceedings of the XXIV International Seminar on Stability Problems for Stochastic Models, Jurmala, Latvia, September $10-17,2004$ 
$\left\{a_{n, i}\right\}_{n \geqslant 1,1 \leqslant i \leqslant k_{n}} \subset \mathbb{R} \backslash\{0\}$ a triangular array of nonzero real constants such that $\max _{1 \leqslant i \leqslant k_{n}}\left|a_{n, i}\right| \rightarrow 0(n \rightarrow \infty)$. Let $\Psi$ be an even, positive, and continuous function such that

$$
\begin{gathered}
\sum_{n=1}^{\infty} \sum_{i=1}^{k_{n}} \frac{\mathrm{E}\left(\Psi\left(\| X_{n, i}||\right)\right)}{\Psi\left(\left|a_{n, i}\right|^{-1}\right)}<\infty, \\
\limsup _{n \rightarrow \infty} \sum_{i=1}^{k_{n}}\left|a_{n, i}\right| \mathrm{E} \| X_{n, i}||<\infty,
\end{gathered}
$$

and

$$
\sum_{n=1}^{\infty}\left(\sum_{i=1}^{k_{n}} a_{n, i}^{2} \mathrm{E}\left\|X_{n, i}\right\|^{2}\right)^{s}<\infty
$$

for some $s>0$. Then convergence of $\prod_{i=1}^{k_{n}} a_{n, i} X_{n, i} \rightarrow 0(n \rightarrow \infty)$ a.s., in $L^{1}$, completely, and in probability are all equivalent.

Proof. The main strategy of proof will be to verify in all four modes of convergence the equivalence of convergence to zero of the products

$$
\prod_{i=1}^{k_{n}} a_{n, i} X_{n, i}
$$

and the sums

$$
\sum_{i=1}^{k_{n}} a_{n, i} X_{n, i}
$$

1. Convergence in $L^{1}$. Suppose (8) converges to zero in $L^{1}$. Put

$$
\sigma_{i, j}:=\left\{\begin{array}{rr}
1, & i<j \\
0, & i=j \\
-1, & i>j
\end{array}\right.
$$

Then we have

$$
\begin{aligned}
& \mathrm{E}\left\|\prod_{i=1}^{k_{n}} a_{n, i} X_{n, i}\right\|=\mathrm{E}\left(\left\|\sum_{i=1}^{k_{n}} a_{n, i} X_{n, i}+\sum_{1 \leqslant i<j \leqslant k_{n}}\left[a_{n, i} X_{n, i}, a_{n, j} X_{n, j}\right]\right\|\right) \leqslant \\
& \leqslant \mathrm{E}\left\|\sum_{i=1}^{k_{n}} a_{n, i} X_{n, i}\right\|+C \cdot \mathrm{E}\left\|\sum_{j=1}^{k_{n}} a_{n, j} X_{n, j} \cdot \sum_{i=1}^{k_{n}} \sigma_{i, j} a_{n, i} X_{n, i}\right\| \leqslant \\
& \leqslant \mathrm{E}\left\|\sum_{i=1}^{k_{n}} a_{n, i} X_{n, i}\right\|+C \sum_{j=1}^{k_{n}}\left|a_{n, j}\right| \mathrm{E}\left\|X_{n, j}\right\| \cdot \mathrm{E}\left\|\sum_{i=1}^{k_{n}} \sigma_{i, j} a_{n, i} X_{n, i}\right\|= \\
&=\mathrm{E}\left\|\sum_{i=1}^{k_{n}} a_{n, i} X_{n, i}\right\|+C \sum_{j=1}^{k_{n}}\left|a_{n, j}\right| \mathrm{E}\left\|X_{n, j}\right\| \cdot \mathrm{E}\left\|\sum_{i=1}^{k_{n}} a_{n, i} X_{n, i}\right\| \rightarrow 0, \\
& n \rightarrow \infty
\end{aligned}
$$

by condition (5) and the symmetry (which allows us to drop the sign $\sigma_{i, j}$ in the last but one line).

Now suppose (7) converges to zero in $L^{1}$ (so, a fortiori, in distribution). By the symmetry of the random variables $X_{n, i}$ and the fact that

$$
(-x) \cdot(-y)=-(y \cdot x) \quad x, y \in G
$$

the reverse products

$$
\prod_{i=1}^{k_{n}} a_{n, k_{n}-i} X_{n, k_{n}-i}
$$

have the same distribution as

$$
-\prod_{i=1}^{k_{n}} a_{n, i} X_{n, i}
$$


so the sequence of the products (9) also converges to 0 in distribution. Due to the fact that

$$
x \cdot y+y \cdot x=2(x+y), \quad x, y \in G,
$$

we have

$$
2 \sum_{i=1}^{k_{n}} a_{n, i} X_{n, i}=\prod_{i=1}^{k_{n}} a_{n, i} X_{n, i}+\prod_{i=1}^{k_{n}} a_{n, k_{n}-i} X_{n, k_{n}-i} .
$$

So (8) converges in distribution to 0 , hence by [2] result (8) converges to 0 in $L^{1}$.

2. Suppose (8) converges in probability to 0 , hence in $L^{1}[2]$. Then we have (for $\varepsilon>0$ ) by Markov's inequality

$$
\mathrm{P}\left(\left\|\prod_{i=1}^{k_{n}} a_{n, i} X_{n, i}\right\| \geqslant \varepsilon\right) \leqslant \frac{1}{\varepsilon} \mathrm{E}\left(\left\|\prod_{i=1}^{k_{n}} a_{n, i} X_{n, i}\right\|\right),
$$

which tends to 0 (as $n \rightarrow \infty$ ) by point 1 .

On the other hand, assume (7) converges in probability to zero. Since the reverse products (9) have the same distributions as minus the products (7), they also converge to zero in probability. Then by writing, as under point 1 , the sum (8) as "product plus reverse product" (as in (10)), it follows that the sums (8) also converge to zero in probability.

3. The proof for complete convergence is analogous to point 2 .

4. Assume (8) converges a.s. to 0 . Then [2] it converges to 0 completely. By point 3 , thus also the products (7) converge to zero completely, hence a.s. On the other hand, if (7) converges to zero a.s., then it converges to zero in probability, hence by point 2, the sums (8) converge to 0 in probability. By [2] it follows that the sums (8) converge a.s. to zero.

\section{REFERENCES}

1. D. Neuenschwander, Probabilities on the Heisenberg Group: Limit Theorems and Brownian Motion, Springer, Berlin (1996).

2. M. Ordóñez Cabrera and S. H. Sung, "On complete convergence of weighted sums of random elements," Stoch. Anal. Appl., 20, No. 1, 21-32 (2002). 